\title{
A BOUNDARY VALUE PROBLEM FOR A NONLINEAR DIFFERENTIAL EQUATION WITH A SMALL PARAMETER
}

\section{EARL A. CODDINGTON AND NORMAN LEVINSON}

It is the purpose of this paper to prove Theorems 1 and 2 below which relate the existence, uniqueness, and general behavior of the solution, $y(x, \epsilon)$, for small $\epsilon>0$, of the two-point boundary value problem

$$
\begin{gathered}
\epsilon y^{\prime \prime}+f(x, y) y^{\prime}+g(x, y)=0, \\
y(0)=y_{0}, \quad y(1)=y_{1},
\end{gathered}
$$

with the solution $u(x)$ of the corresponding "degenerate" initial value problem

$$
f(x, u) u^{\prime}+g(x, u)=0, \quad u(1)=y_{1} .
$$

Theorem 1 (Existence). Let $\left(0, y_{0}\right),\left(1, y_{1}\right)$ be two points in the real $(x, y)$-plane, and assume:

(i) $f(x, y), g(x, y)$ are real functions such that the differential equation

$$
f(x, u) u^{\prime}+g(x, u)=0
$$

has a solution $u(x)$ on $0 \leqq x \leqq 1$, with $u(1)=y_{1}$ and $u(0)=u_{0} \geqq y_{0}$.

(ii) $f(x, y), g(x, y)$ are of class $C^{\prime}$ in a region

$$
R: \quad 0 \leqq x \leqq 1, \quad|y-u(x)| \leqq a, \quad a>0,
$$

which includes the point $\left(0, y_{0}\right)$.

(iii) There exists a constant $k>0$ such that $f(x, y) \geqq k$ for $(x, y)$ in $R$.

Then, for all sufficiently small $\epsilon>0$, there exists in $R$ a solution $y(x)=y(x, \epsilon)$ of

$$
\epsilon y^{\prime \prime}+f(x, y) y^{\prime}+g(x, y)=0
$$

satisfying the boundary conditions

$$
y(0)=y_{0}, \quad y(1)=y_{1} .
$$

Further, $y(x, \epsilon) \rightarrow u(x), y^{\prime}(x, \epsilon) \rightarrow u^{\prime}(x)$, as $\epsilon \rightarrow 0$, uniformly on any subinterval $0<\delta \leqq x \leqq 1$.

Remarks. From the proof of Theorem 1 it will be seen that the

Presented to the Society, October 27, 1951; received by the editors March 28, 1951. 
result of the theorem is valid if the hypotheses (ii) and (iii) are replaced by the somewhat weaker assumptions

(ii) $^{*} f(x, y), g(x, y)$ are of class $C^{\prime}$ in a region

$$
\begin{array}{rrr}
R^{*}: & 0 \leqq x \leqq \alpha<1, \quad y_{0}-\beta \leqq y \leqq u_{0}+\beta & (\alpha, \beta>0), \\
\alpha \leqq x \leqq 1, \quad|y-u(x)| \leqq \gamma & (\gamma>0) ;
\end{array}
$$

(iii)* there exists a constant $k>0$ such that $f(x, u(x)) \geqq k, 0 \leqq x \leqq 1$, and

$$
\int_{y}^{u_{0}} f(0, y) d y>0, \quad y_{0} \leqq y<u_{0}
$$

and if $R$ is replaced by $R^{*}$.

The assumption in (i) that $u_{0} \geqq y_{0}$ is no restriction for if $u_{0}<y_{0}$, then the change $y^{*}=-y, u^{*}=-u$ gives two equations (1)* and (2)* of the same type as (1) and (2) but with $u_{0}^{*}>y_{0}^{*}$. Also, by a change of variable of the type $x^{*}=p x+q$, the interval $0 \leqq x \leqq 1$ can be replaced by an arbitrary bounded interval. It is further clear that if $\epsilon<0$, a similar theorem will hold with the role of the left and right boundaries, $x=0$ and $x=1$, in terchanged.

Assuming the existence of both $u(x)$ and $y(x, \epsilon)$, R. v. Mises [2]1 proved recently that as $\epsilon \rightarrow+0, y(x, \epsilon) \rightarrow u(x)$ and $y^{\prime}(x, \epsilon) \rightarrow u^{\prime}(x)$ uniformly on every subinterval $0<\delta \leqq x \leqq 1$. He assumed that $f(x, y)$, $g(x, y)$ were continuous on a rectangle containing $\left(0, y_{0}\right)$ and $(x, u(x))$, and $f>0$ there.

TheOREM 2 (Uniqueness). Under the assumptions (i), (ii), (iii) of Theorem 1, for sufficiently small $\epsilon>0$, there exists at most one solution $y(x, \epsilon)$ of (2) in $R_{0}$ satisfying the boundary conditions $y(0)=y_{0}, y(1)$ $=y_{1}$. The region $R_{0}$ satisfies the same inequalities as $R$ but with a replaced by a smaller quantity.

REMARK. If (ii) and (iii) are replaced by (ii)* and (iii)* the result of Theorem 2 is valid in $R_{0}^{*}$ where $R_{0}^{*}$ satisfies the same inequalities as $R^{*}$ but with $\beta$ and $\gamma$ replaced by smaller quantities.

Proof of Theorem 1. If $y_{0}<u_{0}$, let $y_{0}^{\prime}>0$. By virtue of (ii) there exists, on a sufficiently small interval to the right of $x=0$, a solution of $(2), y(x)=y(x, \epsilon)=y\left(x, \epsilon ; y_{0}, y_{0}^{\prime}\right)$, for which $y(0, \epsilon)=y_{0}$, $y^{\prime}(0, \epsilon)=y_{0}^{\prime}$, and such that $y(x, \epsilon)$ remains inside $R$. Put

$$
\epsilon y_{0}^{\prime}=\int_{y_{0}}^{u_{0}} f(0, y) d y+\mu,
$$

\footnotetext{
1 Numbers in brackets refer to the references cited at the end of the paper.
} 
where $\epsilon$ is such that $\epsilon^{1 / 2}$ is less than the integral in (3), and $\mu$ is a real parameter whose magnitude is so small that $\epsilon y_{0}^{\prime}>\epsilon^{1 / 2}>0$. It will be shown that given any $\delta_{1}, 0<\delta_{1}<a$, then for small enough $\epsilon$ and $\mu$ there exists a $\xi>0$ such that

$$
\epsilon y^{\prime}(\xi)=\epsilon^{1 / 2}
$$

and if $y(\xi)=\eta$,

$$
0<\xi \leqq\left(u_{0}+\delta_{1}-y_{0}\right) \epsilon^{1 / 2}, \quad\left|\eta-u_{0}\right|<\delta_{1} .
$$

For small enough $\epsilon$, and for $\mu>0$, it will be the case that $\eta>u_{0}$, while for $\mu<0, \eta<u_{0}$. In fact there exists a $\sigma>0$ (independent of $\epsilon$ ) such that for $\mu>0, \eta-u_{0}>\mu \sigma+O\left(\epsilon^{1 / 2}\right)$ and for $\mu<0, u_{0}-\eta>-\mu \sigma+O\left(\epsilon^{1 / 2}\right)$.

In order to prove these facts, let $y^{\prime}(x)=\epsilon^{-1 / 2}$ for the first time at $x=\xi_{1}$, and $y(x)=u_{0}+\delta_{1}$ for the first time at $x=\xi_{2}$, and define $\xi$ $=\min \left(\xi_{1}, \xi_{2}\right)$. For small $\epsilon$ either $\xi_{1}$ or $\xi_{2}$ must exist and thus $\xi$ exists. From the definition of $\xi$, and the mean value theorem for $y(x)$, it follows that $0<\xi \leqq\left(u_{0}+\delta_{1}-y_{0}\right) \epsilon^{1 / 2}$. Integrating (2) from $x=0$ to $x=\xi$, and using (3), we have if $\eta=y(\xi)$

$$
\begin{aligned}
\epsilon y^{\prime}(\xi)= & \int_{\eta}^{u_{0}} f(0, y) d y+\mu \\
& +\int_{0}^{\xi}[f(0, y(x))-f(x, y(x))] y^{\prime}(x) d x-\int_{0}^{\xi} g(x, y(x)) d x .
\end{aligned}
$$

From (ii) it follows that the last two terms are $O\left(\epsilon^{1 / 2}\right)$, as $\epsilon \rightarrow 0$, and hence the above may be written as ${ }^{2}$

$$
\epsilon y^{\prime}(\xi)=\int_{\eta}^{u_{0}} f(0, y) d y+\mu+O\left(\epsilon^{1 / 2}\right) .
$$

For fixed $\delta_{1}>0$ and $|\mu|$ and $\epsilon$ small it follows from (6), since $y^{\prime}(\xi)>0$ and $f\left(0, u_{0}\right)>0$, that $\eta=u_{0}+\delta_{1}$ is impossible. Thus in fact $\xi=\xi_{1}$ and (4) follows. From (6) follows

$$
\int_{\eta}^{u_{0}} f(0, y) d y+\mu=O\left(\epsilon^{1 / 2}\right) .
$$

Moreover (7) implies that if $|\mu|$ and $\epsilon$ are sufficiently small, $\left|\eta-u_{0}\right|$ $<\delta_{1}$. This proves (5). The statements following (5) follow easily from (7) if $\sigma f\left(0, u_{0}\right)=1 / 2$.

If $y_{0}=u_{0},(3)$ becomes $\epsilon y_{0}^{\prime}=\mu$, and if $\mu>\epsilon^{1 / 2}>0$, define $\xi_{1}, \xi_{2}$, and

2 In the following all $O$ terms refer to $\epsilon \rightarrow 0$. 
$\xi$ as before. Then $0<\xi \leqq \delta_{1} \epsilon^{1 / 2}$, and (6) follows as before. If $\mu<-\epsilon^{1 / 2}$. $<0$, define $x=\xi_{1}$ to be the first point such that $y^{\prime}(x)=-\epsilon^{-1 / 2}$, and define $x=\xi_{2}$ to be the first point for which $y(x)=u_{0}-\delta_{1}$. If $\xi$ $=\min \left(\xi_{1}, \xi_{2}\right)$ in this case, then $0<\xi \leqq \delta_{1} \epsilon^{1 / 2}$, and again (6) follows. The remainder of the proof of (4), (5), and the remarks following (5) is the same as for the case $y_{0}<u_{0}$.

Denote by $u(x ; \xi, \eta)$ that solution of (1) for which $u(\xi ; \xi, \eta)=\eta$. This solution will exist on $0 \leqq x \leqq 1$ if $\epsilon$ and $\delta_{1}$ are small enough since the given solution $u(x)$ is a continuous function of its initial point. Choose $\epsilon$ and $\delta_{1}$ so small that this will be true. Then $u(x ; \xi, \eta) \rightarrow u(x)$ uniformly on $0 \leqq x \leqq 1$ as $(\xi, \eta) \rightarrow\left(0, u_{0}\right)$.

It will now be shown that as far to the right of $\xi$ as $y(x, \epsilon), y^{\prime}(x, \epsilon)$ exist

$$
\begin{aligned}
|y(x, \epsilon)-u(x ; \xi, \eta)| & =O\left(\epsilon^{1 / 2}\right), & \xi \leqq x \leqq 1, \\
\left|y^{\prime}(x, \epsilon)-u^{\prime}(x ; \xi, \eta)\right| & =O(\epsilon), & \xi+\epsilon^{1 / 2} \leqq x \leqq 1 .
\end{aligned}
$$

By a familiar continuation argument, this will then prove the existence of $y(x, \epsilon)$ on $0 \leqq x \leqq 1$.

In order to prove (8), let $z(x)=z(x, \epsilon ; \xi, \eta)=y(x, \epsilon)-u(x ; \xi, \eta)$. From (1) and (2), if $\xi \leqq x \leqq 1$, and $y(x, \epsilon)$ exists and is in $R$ (if $x$ is near enough to $\xi, y(x, \epsilon)$ certainly exists), then

$$
\epsilon z^{\prime \prime}+f(x, y) z^{\prime}=r
$$

where

$$
\begin{aligned}
r= & {[g(x, u)-g(x, y)]+[f(x, u)-f(x, y)] u^{\prime} } \\
& +\epsilon f^{-2}(x, u)\left[f(x, u) \frac{\partial g}{\partial x}(x, u)-g(x, u) \frac{\partial f}{\partial x}(x, u)\right] \\
& +\epsilon f^{-3}(x, u)\left[\frac{\partial f}{\partial u}(x, u) g^{2}(x, u)-\frac{\partial g}{\partial u}(x, u) g(x, u) f(x, u)\right] .
\end{aligned}
$$

Because of the assumptions (ii), (iii), and the mean value theorem, it follows that there exists a constant $c>0$ such that

$$
|r| \leqq c[|z|+\epsilon] \text {. }
$$

Integrating (10) we obtain

$$
\begin{aligned}
z^{\prime}(x)= & z^{\prime}(\xi) \exp \left[-\frac{1}{\epsilon} \int_{\xi}^{x} f(t, y(t)) d t\right] \\
& +\frac{1}{\epsilon} \int_{\xi}^{x} r \exp \left[-\frac{1}{\epsilon} \int_{s}^{x} f(t, y(t)) d t\right] d s .
\end{aligned}
$$


Since $y^{\prime}(\xi, \epsilon)=\epsilon^{-1 / 2},\left|z^{\prime}(\xi)\right| \leqq 2 \epsilon^{-1 / 2}$ if $\epsilon$ is sufficiently small, and if this is combined with the fact that $f(x, y) \geqq k$, then (12) gives

$$
\begin{aligned}
\left|z^{\prime}(x)\right| \leqq & 2 \epsilon^{-1 / 2} \exp \left[-\frac{k}{\epsilon}(x-\xi)\right] \\
& +\frac{c \epsilon}{k}+\frac{c}{\epsilon} \int_{\xi}^{x}|z(s)| \exp \left[-\frac{k}{\epsilon}(x-s)\right] d s .
\end{aligned}
$$

Integrating the inequality (13) yields the following estimate for $|z(x)|$ (note that $z(\xi)=0$ ),

$$
\begin{aligned}
|z(x)| \leqq & \frac{2 \epsilon^{1 / 2}}{k}+\frac{c \epsilon}{k} \\
& +\frac{c}{\epsilon} \int_{\xi}^{x}\left(\int_{\xi}^{t}|z(s)| \exp \left[-\frac{k}{\epsilon}(t-s)\right] d s\right) d t
\end{aligned}
$$

and by an interchange of the order of integration in the last term we obtain

$$
|z(x)| \leqq \frac{2 \epsilon^{1 / 2}}{k}+\frac{c \epsilon}{k}+\frac{c}{k} \int_{\xi}^{x}|z(s)| d s .
$$

If $\epsilon$ is small enough, $c \epsilon<\epsilon^{1 / 2}$, and hence

$$
|z(x)| \leqq \frac{3 \epsilon^{1 / 2}}{k}+\frac{c}{k} \int_{\xi}^{x}|z(s)| d s .
$$

It follows easily from this that

$$
\int_{\xi}^{x}|z(s)| d s \leqq \frac{3 \epsilon^{1 / 2}}{c}\left[\exp \left(\frac{c}{k}(x-\xi)\right)-1\right], \quad \xi \leqq x \leqq 1,
$$

and therefore

$$
|z(x)| \leqq \frac{3 \epsilon^{1 / 2}}{k} \exp \left(\frac{c}{k}(x-\xi)\right), \quad \xi \leqq x \leqq 1 .
$$

This proves the estimate (8). Formula (9) follows directly from (8) and (13).

It still remains to show that $y_{0}^{\prime}$ can be chosen so that $y(1, \epsilon)=y_{1}$. From the remark after (5), if $\mu<0$ and $\epsilon$ is small enough then $u_{0}-\eta$ $>-\mu \sigma / 2$. Hence by the continuity of $u(x ; \xi, \eta)$ in $\xi$ and uniqueness, $u(x ; \xi, \eta)<u(x)$, and on account of $(8), y(x, \epsilon)<u(x)$ on $\xi \leqq x \leqq 1$ if $\epsilon$ is sufficiently small. In particular $y(1, \epsilon)<y_{1}$. Similarly, for $\mu>0$, and $\epsilon$ small enough, $y(1, \epsilon)>y_{1}$. Since $y(x, \epsilon)$ is in $R$ for $0 \leqq x \leqq 1$ 
for small $\epsilon$ and $\mu, y(x, \epsilon)$ is continuous in $\mu$. By the continuity of $y(1, \epsilon)$ with respect to $\mu$ it follows that for $\epsilon$ sufficiently small, and some $\mu, y(1, \epsilon)=y_{1}$. This, with (8) and (9), completes the proof of the existence theorem for the boundary value problem.

Proof of Theorem 2. It will be shown that the solution $y(x, \epsilon)$ $=y\left(x, \epsilon ; y_{0}, y_{0}^{\prime}\right)$ considered as a function of $y_{0}^{\prime}$ satisfies

$$
\frac{\partial y}{\partial y_{0}^{\prime}}\left(1, \epsilon ; y_{0}, y_{0}^{\prime}\right)>0,
$$

for $\epsilon$ sufficiently small. This clearly implies the uniqueness of $y(x, \epsilon)$ satisfying (2) and $y(0, \epsilon)=y_{0}, y(1, \epsilon)=y_{1}$.

Let

$$
w(x)=w\left(x, \epsilon ; y_{0}, y_{0}^{\prime}\right)=\frac{\partial y}{\partial y_{0}^{\prime}}\left(x, \epsilon ; y_{0}, y_{0}^{\prime}\right) .
$$

For fixed $\epsilon, w(x), w^{\prime}(x), w^{\prime \prime}(x)$ exist for $0 \leqq x \leqq 1,{ }^{3}$ and from (2)

$$
\epsilon w^{\prime \prime}+f w^{\prime}+\left(\frac{\partial f}{\partial y} y^{\prime}+\frac{\partial g}{\partial y}\right) w=0 .
$$

It is clear that

$$
w(0)=0, \quad w^{\prime}(0)=1 .
$$

In order to prove (14) we first obtain appraisals for $w(x), w^{\prime}(x)$ near $x=0$. If (15) is integrated, the initial values (16) being used, then this yields

$$
\epsilon w^{\prime}+f w=\epsilon+\int_{0}^{x}\left(\frac{\partial f}{\partial x}-\frac{\partial g}{\partial y}\right) w d t .
$$

Another integration results in the following expression for $w(x)$ :

$$
\begin{aligned}
w(x)= & \int_{0}^{x} E(s, x ; \epsilon) d s \\
& +\frac{1}{\epsilon} \int_{0}^{x}\left(\frac{\partial f}{\partial x}-\frac{\partial g}{\partial y}\right) w\left[\int_{r}^{x} E(s, x ; \epsilon) d s\right] d r,
\end{aligned}
$$

where

$$
E(s, x ; \epsilon)=\exp \left(-\frac{1}{\epsilon} \int_{0}^{x} f d t\right) .
$$
[1].

${ }^{3}$ This follows from an application of a well known theorem to (2); see, for example, 
By (ii), there exists a constant $m>0$ such that

$$
\left|\frac{\partial f}{\partial x}(x, y(x))-\frac{\partial g}{\partial y}(x, y(x))\right| \leqq m, \quad 0 \leqq x \leqq 1,
$$

and from (iii), $f(x, y(x)) \geqq k>0$ on $0 \leqq x \leqq 1$. These facts, together with (18), imply

$$
|w(x)| \leqq \frac{\epsilon}{k}+\frac{m}{k} \int_{0}^{x}|w(s)| d s,
$$

and this in turn implies

$$
\int_{0}^{x}|w(s)| d s \leqq \frac{\epsilon}{m}\left[\exp \left(\frac{m x}{k}\right)-1\right] .
$$

If (21) and (19) are applied to (18), there results

$$
w(x)=\int_{0}^{x} E(s, x ; \epsilon) d s+x O(\epsilon) .
$$

This can be written as

$$
\begin{aligned}
w(x)= & \frac{1}{F(x)} \int_{0}^{x} F(s) E(s, x ; \epsilon) d s \\
& +\frac{1}{F(x)} \int_{0}^{x}[F(x)-F(s)] E(s, x ; \epsilon) d s+x O(\epsilon),
\end{aligned}
$$

where $F(x)=f(x, y(x))$.

We appraise the second integral on the right in (22). Let $\rho=\xi+\epsilon^{1 / 2}$, and suppose $x \geqq \rho+\epsilon^{1 / 2}$. By splitting the integral into two parts $I_{1}$ and $I_{2}$, where $I_{1}$ is the integral from 0 to $\rho, I_{2}$ the integral from $\rho$ to $x$, we have first of all

$$
I_{1} \leqq 2 M \int_{0}^{\rho} e^{-k / e^{1 / 2}} d s \leqq M e^{-k / \epsilon^{1 / 2}},
$$

where $M=\max |f(x, y)|$ on $R$, and therefore $I_{1} / F(x)=O\left(\epsilon^{2}\right)$. Since both $x, s \geqq \xi+\epsilon^{1 / 2}$ in $\rho \leqq x \leqq 1$, it follows from (ii) and (9) that $F(x)$ satisfies a Lipschitz condition in $\rho \leqq x \leqq 1$. Thus

$$
I_{2} / F(x)=O\left(\int_{p}^{x}(x-s) E(s, x ; \epsilon) d s\right),
$$

and by a simple calculation it is seen that this implies $I_{2} / F(x)$ $=O\left(\epsilon^{2}\right)$. 
Therefore, after integrating the first integral on the right of (22), we obtain

$$
w(x)=\frac{\epsilon}{F(x)}[1-E(0, x ; \epsilon)]+O\left(\epsilon^{2}\right)+x O(\epsilon), \quad x \geqq \xi+2 \epsilon^{1 / 2} .
$$

From (17) it now follows that

$$
w^{\prime}(x)=O(\epsilon+x), \quad x \geqq \xi+2 \epsilon^{1 / 2} .
$$

Suppose $\lambda<0$, and $v(x)$ is defined by the relation

$$
w(x)=v(x) \exp \left(\frac{\lambda x}{\epsilon}\right) .
$$

Clearly

$$
w^{\prime}(x)=\frac{\lambda}{\epsilon} w(x)+v^{\prime}(x) \exp \left(\frac{\lambda x}{\epsilon}\right) .
$$

Then from (23) and (24), for small $x_{1} \geqq \xi+2 \epsilon^{1 / 2}$,

$$
v\left(x_{1}\right)>0, \quad v^{\prime}\left(x_{1}\right)>0 .
$$

Now (15) implies that $v(x)$ satisfies the equation

$$
\epsilon v^{\prime \prime}+(f+2 \lambda) v^{\prime}+\left(\frac{\lambda^{2}}{\epsilon}+\frac{\lambda f}{\epsilon}+\frac{\partial f}{\partial y} y^{\prime}+\frac{\partial g}{\partial y}\right) v=0 .
$$

It follows from (9) that $y^{\prime}$ is bounded for $x_{1} \leqq x \leqq 1$; and by (ii), the same is true for $\partial f / \partial y, \partial g / \partial y$. Thus there exists a constant $m *>0$ such that

$$
\left|\frac{\partial f}{\partial y} y^{\prime}+\frac{\partial g}{\partial y}\right|<m *, \quad x_{1} \leqq x \leqq 1 .
$$

Let $x=x_{2}, x_{1}<x_{2} \leqq 1$, be the first point to the right of $x=x_{1}$ where $v^{\prime}\left(x_{2}\right)=0$. From (26) and (27) it is clear that $\epsilon^{2} v^{\prime \prime}\left(x_{2}\right)$ has the same sign as $-\left(\lambda^{2}+\lambda f\right) v\left(x_{2}\right)$ if $\epsilon$ is sufficiently small. But $v\left(x_{2}\right)>0$ by (25), and $\lambda<0$ can be chosen so that $\lambda^{2}+\lambda f<0$, since $f\left(x_{2}, y\left(x_{2}\right)\right)>0$. Hence $v^{\prime \prime}\left(x_{2}\right)>0$ if $\epsilon$ is sufficiently small. However this implies that $v^{\prime}(x)$ is increasing at $x=x_{2}$, which contradicts the fact that $v^{\prime}(x)>0$, $x_{1} \leqq x<x_{2}, v^{\prime}\left(x_{2}\right)=0$. Therefore $v^{\prime}(x)>0$ for $x_{1} \leqq x \leqq 1$, and since $v\left(x_{1}\right)$ $>0$, it follows that $v(x)>0$ for $x_{1} \leqq x \leqq 1$. Thus $w(x)>0, x_{1} \leqq x \leqq 1$, and in particular $w(1)=\partial y / \partial y_{0}^{\prime}>0$ at $x=1$. This proves (14), and hence the uniqueness theorem for $\mu$ sufficiently small.

For the case $\mu$ not small we note that the only use of Theorem 1 
in the above proof was in the application of formula (9). If $y(x, \epsilon)$ is any solution of (2) remaining in $R_{0}$, then we show (9) must hold. There are two cases, either $\left|y_{0}^{\prime}\right| \leqq \epsilon^{-1 / 2}$ or $\left|y_{0}^{\prime}\right|>\epsilon^{-1 / 2}$. For the latter situation it is clear that $y(x, \epsilon)$ cannot remain in $R_{0}$ for small $\epsilon$ unless there exists a $\xi, 0<\xi<1$, such that $\left|y^{\prime}(x, \epsilon)\right|=\epsilon^{-1 / 2}$ for the first time at $x=\xi$. If $\xi$ exists, then it follows by the mean value theorem that $\xi=O\left(\epsilon^{1 / 2}\right)$. In either situation there exists a $\xi$ for which $0 \leqq \xi<1$, $\xi=O\left(\epsilon^{1 / 2}\right),\left|y^{\prime}(\xi, \epsilon)\right| \leqq \epsilon^{-1 / 2}$. The existence of such a $\xi$ is all that is needed to prove (8), (9) for the solution of (1), $u(x ; \xi, \eta)$, which passes through $\xi, \eta=y(\xi, \epsilon)$ and which remains in $R$. But since $y(x, \epsilon)$ remains in $R_{0}$, it follows from (8) that $u(x ; \xi, \eta)$ remains in $R$ for $\epsilon$ sufficiently small. Therefore the argument used for $\mu$ small can be applied to extend the uniqueness to any solution $y(x, \epsilon)$ remaining in $R_{0}$.

For the more general case $\epsilon y^{\prime \prime}+F\left(x, y, y^{\prime}\right)=0$ the results of Theorems I and II are not valid unless $F$ is severely restricted. Thus even $F=y^{\prime}+\left(y^{\prime}\right)^{3}$ is not restricted enough for Theorem $\mathrm{I}$ to hold as can be seen by direct integration.

\section{REFERENCES}

1. E. Kamke, Differentialgleichungen reeller Funktionen, Leipzig, 1930, p. 155.

2. R. v. Mises, Die Grenzschichte in der Theorie der gewöhnlichen Differentialgleichungen, Acta Univ. Szeged., Leopoldo Fejer er Frederico Riesz LXX annos natis dedicatus, Pars B, vol. 12 (1950) pp. 29-34.

Massachusetts Institute of TeChNoLogy 